\title{
Belgeo
}

Revue belge de géographie

\section{Cotation des revues de géographie, impérialisme scientifique anglo-saxon et culture de l'excellence marchandisée}

Rankings of geographical journals, Anglo-Saxon scientific imperialism, and merchandised scientific excellence

\section{Christian Vandermotten}

\section{CpenEdition}

\section{Journals}

Édition électronique

URL : http://journals.openedition.org/belgeo/7131

DOI : 10.4000/belgeo.7131

ISSN : 2294-9135

Éditeur :

National Committee of Geography of Belgium, Société Royale Belge de Géographie

\section{Référence électronique}

Christian Vandermotten, « Cotation des revues de géographie, impérialisme scientifique anglo-saxon et culture de l'excellence marchandisée », Belgeo [En ligne], 1-2 | 2012, mis en ligne le 04 décembre 2012, consulté le 01 mai 2019. URL : http://journals.openedition.org/belgeo/7131 ; DOI : 10.4000/ belgeo.7131

Ce document a été généré automatiquement le 1 mai 2019.

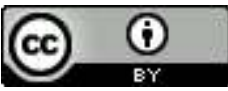

Belgeo est mis à disposition selon les termes de la licence Creative Commons Attribution 4.0 International. 


\title{
Cotation des revues de géographie, impérialisme scientifique anglo-saxon et culture de l'excellence marchandisée
}

\author{
Rankings of geographical journals, Anglo-Saxon scientific imperialism, and \\ merchandised scientific excellence
}

Christian Vandermotten

\section{Introduction}

1 Les impacts sur la production géographique de la domination scientifique et idéologique anglo-saxonne, à travers le véhicule de l'usage international de l'anglais, mais aussi du quasi-monopole sur la littérature dominante qu'il accorde aux éditeurs commerciaux anglo-saxons (ou assimilés, comme le néerlandais Elsevier) ont été soulignés dans l'Editorial du présent numéro de Belgeo. La plupart des journaux de géographie listés dans le classement du Web of Science sont toujours publiés dans des pays anglo-saxons. Les comités éditoriaux de ces revues sont majoritairement formés de géographes anglosaxons, aux normes desquelles la formatisation des articles est soumise.

La question d'une critique des normes d'appréciation des revues de géographie est donc d'actualité, au regard de cet impérialisme réducteur. L'exercice est toutefois délicat, puisqu'il s'agit de ne pas tomber à son tour dans le piège des classements, même alternatifs. Tentons de rester à l'objectif d'établir un inventaire des revues géographiques de qualité et qui peuvent intéresser un nombre important de géographes, en gardant autant que faire se peut un équilibre entre la littérature francophone (ou en d'autres langues) et anglophone.

Dassa, Kosmopoulos et Pumain (2010) se sont récemment essayées à établir une analyse des bases de données scientifiques internationales en sciences humaines et sociales, en se limitant à établir une comparaison des revues "reconnues" par différentes listes faisant 
autorité, mais sans tenir compte des "cotations" de ces revues. Leurs conclusions soulignaient la très imparfaite concordance de ces listes et, sans surprise, la surreprésentation évidente des revues anglo-saxonnes (et éditées par les grands éditeurs commerciaux internationaux) et la couverture plus large assurée par SCOPUS que par le Web of Science.

Par rapport au travail de Dassa, Kosmopoulos et Pumain, nous réduirons ici le champ au seul domaine de la géographie, mais en élargissant l'investigation aux cotations des revues.

Plus encore que dans d'autres disciplines, la délimitation du champ des titres à considérer pour réaliser un état des revues de géographie (et de celles qui peuvent intéresser des géographes, tant physiciens que sociaux) est délicate. Les raisons de ces difficultés vont jusqu'à poser la question, régulièrement débattue, de l'existence même de la géographie en tant que champ disciplinaire pertinent.

6 Sachant ces problèmes et le flou des classements et rattachements disciplinaires dans les grandes bases de données bibliographiques, avec des recouvrements de discipline et doublons nombreux, nous avons procédé, pour établir la liste des revues de géographie, de manière empirique: sont considérées comme revues de géographie celles qui sont dépouillées comme telles dans au moins une des bases de données examinées ${ }^{1}$.

7 Côté francophone, la Bibliographie géographique internationale (BGI) a dépouillé en 2011150 revues possédant un numéro ISSN2². Malgré ses objectifs d'exhaustivité, la BGI reste très soumise, dans la sélection des revues dépouillées, aux disponibilités résultant d'échanges, de sorte qu'elle couvre moins bien les revues internationales (anglo-saxonnes) éditées par les éditeurs commerciaux.

8 Pour sa part, l'Agence pour l'Évaluation de la Recherche en France (AERES), dont les listes font référence pour déterminer les revues qualifiantes pour les candidats à des postes académiques dans ce pays, reprend 232 revues pour le domaine de la géographie. Parmi celles-ci, 74 seulement sont communes avec celles examinées par la BGI.

La BGI n'a évidemment pas pour mission de donner une cote aux revues. Quant à l'AERES, elle a renoncé à hiérarchiser les revues reprises dans sa liste au-delà de la reconnaissance d'une qualité suffisante par ce seul fait, justement pour éviter de sacrifier au culte du ranking.

Côté anglo-saxon, la base SCOPUS, publiée par Elsevier, recense 446 revues dans sa rubrique "Geography, planning and development". Les bases SCIE (Sciences exactes et naturelles) et SOCS (Sciences sociales) du Web of Science, publié par Thompson Reuters, sont beaucoup plus restrictives, puisqu'elles ne retiennent que 103 revues dans les rubriques Geography, physical sciences d'une part, Geography, social sciences, de l'autre.

11684 revues sont ainsi considérées comme des revues de géographie par au moins une des quatre sources.

\section{L'open access}

On peut y ajouter des revues en open access recensées dans le Directory of Open Access Journals $(D O A J)^{3}: 100$ revues y sont reprises dans la rubrique Géography et 49 autres titres peuvent intéresser les géographes, auxquels il faut ajouter 14 revues en open access pour le lecteur, mais payantes pour les auteurs ${ }^{4}$. Certains de ces titres sont aussi repris dans les classements précédents. 
14 Au total, l'ensemble de notre corpus concerne finalement 787 revues, parmi lesquelles 163 en open access.

Ce nombre est du même ordre de grandeur que celui obtenu par Dassa, Kosmopoulos et Pumain (2010, § 48), qui recensent 683 revues dans leur rubrique Géographie (d'autres références, que nous avons retenues comme telles en fonction des critères de sélection explicités ci-dessus, se trouvant reprises par ces auteures dans des rubriques comme la démographie, les sciences de l'environnement, etc.).

Dans ce corpus de 787 revues, 278 bénéficient d'une cotation SCIE ou SOCS dans le Web of Science et 606 dans la base SCOPUS. Seules 9 revues recensées dans SCIE/SOCS ne le sont pas également par SCOPUS, qui apparaît donc définitivement comme un outil bien plus efficace pour le géographe que le Web of Science. Ce corpus de 787 revues sur lequel nous travaillerons est encore bien réduit par rapport à la très large Geobase d'Elsevier, qui recense de l'ordre de 2000 revues actives pouvant intéresser le géographe, voire aux 1310 revues reprises dans le site de l'UGI, ceci traduisant évidemment à nouveau les contours extrêmement larges et flous du champ de la discipline ${ }^{5}$.

\section{Le recensement des revues selon leur nationalité et le bénéfice de l'open access}

17 Le classement des revues par origine nationale n'est pas toujours évident, en particulier pour celles publiées par les grands éditeurs commerciaux internationaux. Nous les avons en général rattachées aux pays d'implantation principale de ces éditeurs, essentiellement le Royaume-Uni, les Pays-Bas, les États-Unis et dans une moindre mesure l'Allemagne. Nous avons néanmoins procédé à quelques écarts par rapport à cette règle : ainsi nous avons considéré le Norsk Geografisk Tidsskrift, journal historique de la Société de Géographie norvégienne, comme une revue norvégienne, même si elle est aujourd'hui publiée par Taylor and Francis.

18 Le biais en faveur des revues en anglais publiées par les éditeurs commerciaux apparaît à l'évidence de manière flagrante : dans l'ensemble des revues recensées par SCOPUS et Web of Science, près des deux-tiers (63,7\%) sont publiées dans les îles britanniques, aux ÉtatsUnis ou aux Pays-Bas (plus de $70 \%$ si on y ajoute l'Allemagne); seuls $4,4 \%$ sont publiées en France. En revanche, si on considère les revues reprises soit par la BGI, soit par l'AERES, les revues françaises représentent $19,8 \%$ du total, presque autant que les britanniques $(24,0 \%)$. L'ensemble des revues britanniques, états-uniennes et néerlandaises ne totalise plus que $49,6 \%$ du total. Il est peu probable que de tels écarts puissent être attribués à une réelle différence objective de qualité de la production scientifique, d'ailleurs bien difficile à déterminer puisqu'elle l'est à ce jour à travers ce système de citations qui autoreproduit partiellement ses résultats. Ajoutons que tant les relevés anglo-saxons que les relevés français sous-estiment grandement la production scientifique du reste du monde : si la part de l'Afrique ( $1 \%$ ou moins) reflète certainement les énormes déficits de ce continent et surtout la difficulté qui y prévaut d'assurer un minimum de continuité aux revues, il est évident que les chiffres concernant le Japon et la Corée ( $0,6 \%$ des revues dans les relevés français, $1,3 \%$ dans les relevés anglo-saxons), la Chine (respectivement $0,6 \%$ et $1,0 \%)^{6}$ ou la Russie $(1,3 \%$ et $0,5 \%)$ ne rendent en rien compte de la quantité et de la qualité de la production scientifique dans ces pays. 
Tableau 1. Répartition des revues recensées selon leur nationalité et le bénéfice de l'open access.

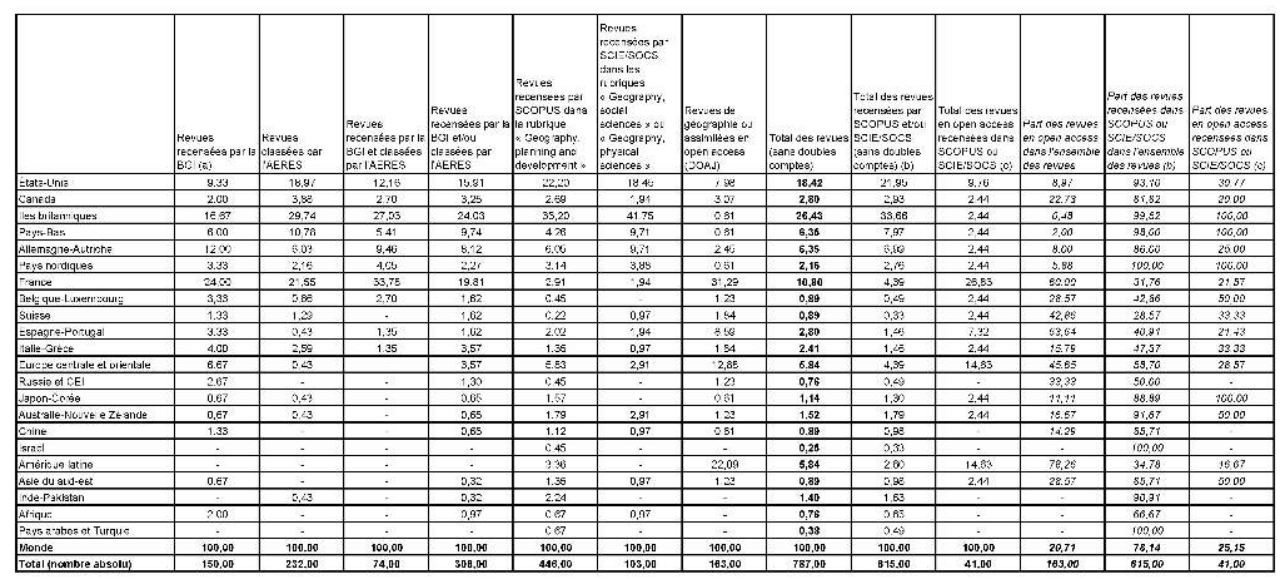

(a) A l'exclusion de "La géographie", devenue "Bulletin de liaison des membres de la Société de géographie de Paris", qui n'est plus une revue scientifique

(b) Quelle que soit la rubrique où elles apparaissent. Parmi ces revues, seules 9 parmi celles recensées par SCIE/SOCS ne le sont pas par SCOPUS (3 britanniques, 2 françaises, 1 autrichienne, 1 canadienne, 1 états-unienne, 1 italienne)

(c) Alors que SCOPUS en recense 40, Web of Science ne recense que 5 revues en open access (une allemande, une slovène, une espagnole, une suisse et une britannique, cette dernière étant la seule à ne pas être reprise aussi dans SCOPUS)

19 La répartition des revues en open access selon leur nationalité présente un contraste saisissant avec le classement global. Le libre accès est presque absent dans les pays où la diffusion de la production scientifique est contrôlée par les grandes maisons d'édition internationales : Îles britanniques, Pays-Bas et, dans une mesure un peu moindre, ÉtatsUnis. Encore les rares journaux en open access dans ces pays imposent-ils souvent une redevance aux auteurs. En revanche, le libre accès est massivement répandu en France, où il est d'ailleurs soutenu par le CNRS qui y trouve sans doute une opportunité de lutte contre le monopole scientifique anglo-saxon (31,3\% de l'ensemble des revues recensées en open access sont françaises). Le libre accès s'est aussi bien répandu dans la péninsule ibérique ( $8,6 \%$ du total), vraisemblablement pour des raisons similaires. Il est probable que la très bonne diffusion du libre accès en Amérique latine (22,1 \% du total des titres), en particulier au Brésil, et dans certains pays d'Europe centre-orientale (12,9\%), surtout en Roumanie, traduise aussi le jeu de réseaux d'influence diplomatico-scientifiques qui visent à contrer la domination anglo-saxonne, dans des pays dont la latinité favorise la diffusion ou du moins le maintien d'une certaine influence et coopération française (le libre accès est d'ailleurs aussi relativement bien implanté au Québec).

\section{Les catégories de revues selon leur spécialisation}

Les 787 revues analysées ont été réparties de manière exclusive entre quelques grandes catégories, avec ici aussi quelques difficultés d'attribution, qui ne remettent néanmoins pas en question les conclusions générales que l'on peut tirer (tableau 2). 
Tableau 2. Répartition des revues recensées selon leur domaine.

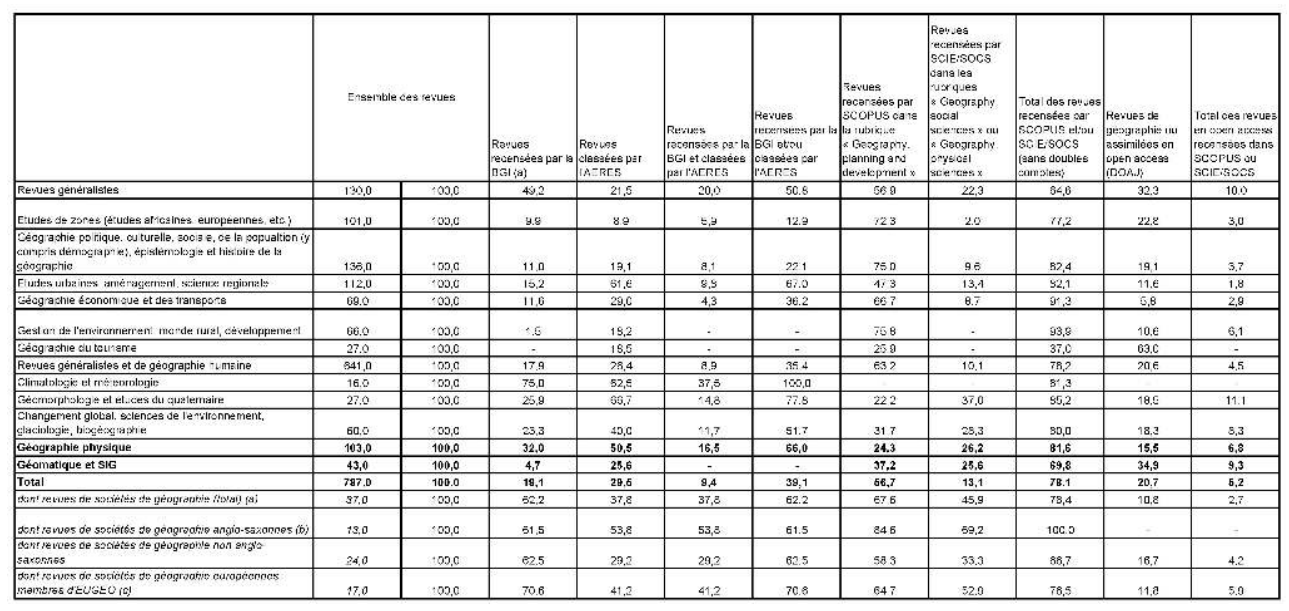

(a) Ont été incluses des revues d'associations de géographes (en France ou en Espagne), ainsi qu'une revue comme Geographia Helvetica, même si elle n'émane pas formellement d'une société suisse de géographie. Le statut de certaines revues de sociétés anglo-saxonnes ou nordiques est ambigu, parce que si elles restent formellement des revues de sociétés de géographie ou portant le label de celles-ci, elles sont de fait devenues des revues publiées par des éditeurs commerciaux. Toutes les revues de cette rubrique entrent dans la catégorie des revues généralistes, à l'exception de Geografiska Annaler, publié sous les auspices de la société suédoise d'anthropologie et de géographie, scindée en une revue de géographie humaine et une autre de géographie physique, et d'un bulletin spécialisé en géomatique publié par une section de l'association des géographes espagnols.

(b) Y compris le Canadian Geographer/Le géographe canadien, non compris les revues de sociétés de pays nordiques rédigées en anglais.

(c) Österreichische Geografische Gesselschaft (Mitteilungen der Österreichischen Geografische Gesellschaft) ; Société Royale Belge de Géographie (BELGEO) ; Czech Geographical Society (Geografie) ; Royal Danish Society (Geografisk Tidsskrift) ; Geographical Society of Finland (Fennia, Terra) ; Hungarian Geographical Society (Foldrajzi kozlemenyek); Geographical Society of Ireland (Irish Geography) ; Societa Geografica Italiana (Bolletino della Societa Geografica Italiana) ; Societa di studi geografici (Rivista Geografica Italiana); Royal Geographical Society (Area; Transactions of the Institute of British Geographers ; Geographical Journal); Asociacion de Geografos Espanoles (Boletin de la Asociacion de Geografos Espanoles ; Geofocus) ; Swedish Anthropological and Geographical Society (Geografiska Annaler, Series A and B).

21 La très grande majorité des revues sont orientées vers la géographie humaine, mais les revues généralistes, jadis typiques de la géographie, et parmi elles les revues des sociétés de géographie, sont devenues fort minoritaires par rapport aux revues spécialisées. Remarquons aussi que, malgré l'importance acquise par cette spécialisation, les revues de géomatique et sur les systèmes d'information géographiques ne représentent qu'à peine plus de $5 \%$ du nombre total de revues.

\section{Les cotations des revues}

Côté francophone, aucune des deux bases considérées n'introduit de cotation. Le biais anglo-saxon sera donc renforcé dans l'analyse qui suit. Notre objectif n'est évidemment pas de réaliser des analyses bibliométriques fines, puisque nous voulons souligner combien elles sont sujettes à caution et en réalité subjectives, mais plutôt de procéder à une large comparaison des revues et à une analyse statistique globale de leurs "reconnaissances" en fonction des thèmes qu'elles traitent, de leur statut éditorial (en particulier l'opposition entre revues "commerciales" et revues des sociétés savantes), de leur éventuel accès en open access. Nous pourrons donc nous contenter de quantifier la position de chaque revue par un indicateur unique simple dans chaque classement, sans 
entrer dans une discussion approfondie des qualités respectives des différents indicateurs bibliométriques proposés.

Nous disposons pour SCOPUS (relevé 2011) du H-index et pour le Web of Science de l'impact factor sur 5 ans (période 2006-2010) ${ }^{7}$. De manière à vérifier l'impact du biais non seulement anglo-saxon mais aussi lié au corpus à partir duquel ces indices sont calculés, tant par SCOPUS que par le Web of Science, nous avons également relevé, pour toutes les revues analysées, le H-index donné pour la même période 2006-2010 par le site bibliométrique d'accès gratuit Publish or Perish. Ce relevé, initié par la chercheuse australienne Anne-Wil Harzing en 1999, se veut une alternative ouverte à l'analyse de l'ensemble de la littérature, sans biais liés à la sélection des revues considérées, puisqu'il examine systématiquement les citations enregistrées sur Google Scholar, c'est-à-dire aussi dans des ouvrages ou des revues non référencées par les deux grandes bases de données bibliométriques. Malgré cette plus grande ouverture, un double biais linguistique subsiste cependant en faveur de l'anglais, d'une part parce qu'il semble que Google Scholar couvre mieux la production en anglais que dans d'autres langues, d'autre part parce que l'écriture en anglais favorise évidemment la citation internationale par rapport à une notoriété éventuellement importante mais plus locale, qui peut caractériser certains travaux géographiques.

L'analyse faite de ces indices ne considère ici que les seuls H-index de Publish or Perish, puisque c'est le site qui fournit la couverture la plus large (tableau 3). Il y a d'ailleurs une excellente corrélation entre les valeurs moyennes de cet indicateur et celles fournies par SCOPUS ou par le Web of Science pour les seules revues classées également par ces deux bases de données ${ }^{8}$. Évidemment, plus la base de données est restreinte (et anglo-saxonne), plus le H-index est élevé : il est de 12,7 en moyenne pour l'ensemble des 761 revues retrouvées dans Publish or Perish, de 15,0 pour les 593 recensées aussi par SCOPUS et de 25,6 pour les 197 reprises dans le Web of Science.

La cotation est plus élevée en moyenne pour les revues de géographie physique, moins nombreuses $(19,9)$ que pour les revues généralistes et de géographie humaine $(11,6)^{9}$, ce qui traduit à la fois le plus grand ancrage des revues de géographie humaine dans le local et les scores moindres des revues de sciences sociales en général. Il est plus étonnant de constater que le score moyen des revues de géomatique n'est que du même ordre que celui des revues généralistes et de géographie humaine $(12,0)$.

Le double biais en faveur de l'anglais et des revues publiées par les grandes maisons d'édition internationales assure une position dominante au classement de trois pays : les Pays-Bas $(27,3)$, la Grande-Bretagne $(18,7)$ et les États-Unis $(15,4)$. On ne peut évidemment prétendre que cela traduise une différence de qualité objective d'une telle ampleur par rapport à la production géographique d'un pays comme la France $(4,4)^{10}$.

Les revues des sociétés de géographie souffrent de la concurrence des revues commerciales ${ }^{11}$. Leur H-index moyen est de 9,4 (15,9 pour les sociétés anglo-saxonnes, seulement 6,1 pour les autres).

L'open access pourrait sembler ne pas entraîner une augmentation évidente de la visibilité, puisque le H-index moyen des revues publiées sous cette forme n'est que de 4,6. Il faut toutefois relativiser ce constat: tout d'abord, beaucoup d'entre elles sont récentes, $\mathrm{du}$ moins sous cette formule; d'autre part, elles sont pour la plupart issues de quatre pays non anglo-saxons, la France, l'Espagne, le Brésil et la Roumanie. Dès lors, il apparaît que leur cotation moyenne est du même ordre que celle de l'ensemble des revues publiées 
dans ces pays $(3,7$, au lieu de 4,0$)$ et que sans doute l'absence de recours à l'open access aurait signifié pour bien de ces revues la disparition ou la non-existence, ce qui aurait fortement limité l'expression de la recherche géographique dans ces pays.

Tableau 3. H-index moyen des revues par catégorie (d'après les données de Publish or Perish).

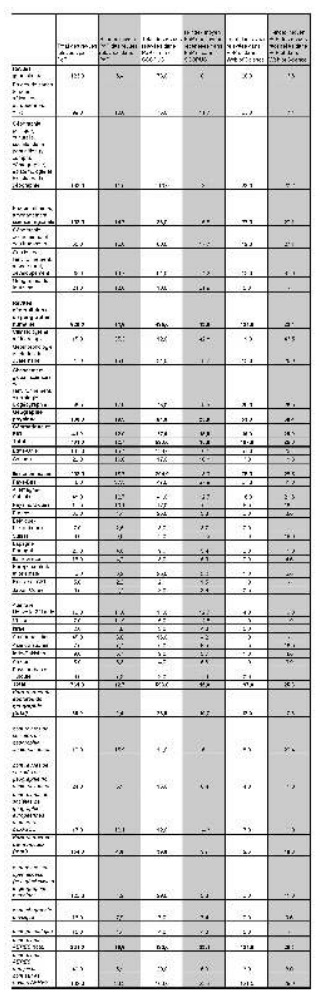

\section{Les préoccupations parallèles de l'Union Géographique Internationale}

Les bases de données qui fondent ce texte étaient déjà traitées à la fin août 2012, au moment de la tenue à Cologne du Congrès de l'UGI. Cette organisation a présenté pour l'occasion une remarquable base de données des revues géographiques mondiales, qui devrait devenir un outil majeur pour la recherche géographique et les étudiants dans cette discipline, puisqu'elle se veut la plus exhaustive possible, évolutive et en outre donne accès à toute la production mise librement en ligne (tables des matières, résumés ou articles). La présentation de ce travail a permis à Ton Dietz, un des maîtres d'œuvre, de présenter des constats qui convergent vers ceux présentés ci-dessus.

Au départ d'une définition large de la littérature géographique et sur la base de recours aux informations collectées dans les différents pays membres, le site de l'UGI, qui ne demande qu'à améliorer encore son recensement, relève à ce jour 1310 revues de géographie. Le recensement, résumé dans le tableau 4 illustre de manière remarquable la sous-valorisation de la littérature non publiée en anglais, ni en Amérique du nord ou dans les pays anglo-saxons européens, et en particulier l'oubli presque total de l'abondante production géographique chinoise. 
$31 \mathrm{Mu}$ lui aussi par une réflexion sur la nécessité de battre en brèche les hégémonies de la littérature anglo-saxonne commercialisée et les biais introduits par des procédures de référencement contrôlées par une ou deux firmes qui sont à la fois juges et parties, Ton Dietz a terminé sa présentation en se demandant si les géographes devaient refuser de publier dans des revues qui ne soient pas en ligne, voire en libre accès.

Tableau 4. Comparaison entre le recensement des revues géographiques (au sens large) par l'UGI, le Web of Science et notre relevé.

\begin{tabular}{|c|c|c|c|c|c|}
\hline $\begin{array}{c}\text { Continents, } \\
\text { pays et langues }\end{array}$ & 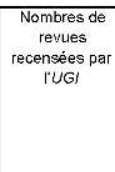 & \begin{tabular}{|l|} 
Part de celles-ci \\
recensees par le \\
Web of Science
\end{tabular} & \begin{tabular}{|c|} 
Répartitition \\
régionale et \\
linguistique des \\
revues \\
recensées par \\
rUG/
\end{tabular} & $\begin{array}{c}\text { Répartition } \\
\text { régionale et } \\
\text { linguistique des } \\
\text { revues } \\
\text { recensées } \\
\text { simultanément } \\
\text { par l'UG let le } \\
\text { Web of Science }\end{array}$ & \begin{tabular}{|c|} 
Répartitition \\
régionale et \\
linguistique des \\
revues reprises \\
dans notre \\
relevé
\end{tabular} \\
\hline Europe & 463 & $54 \%$ & $35 \%$ & $45 \%$ & $66 \%$ \\
\hline $\begin{array}{l}\text { dont Royaume- } \\
\text { Uni }\end{array}$ & 143 & $\ldots$ & $11 \%$ & $\ldots$ & $26 \%$ \\
\hline dont Pays-Bas & 92 & $\ldots$ & $7 \%$ & $\ldots$ & $6 \%$ \\
\hline dont France & 51 & $\ldots$ & $4 \%$ & $\ldots$ & $11 \%$ \\
\hline dont Espagne & 42 & $\ldots$ & $3 \%$ & $\ldots$ & $2 \%$ \\
\hline dont Allemagne & 32 & $\ldots$ & $2 \%$ & $\ldots$ & $6 \%$ \\
\hline dont Belgique & 4 (a) & $\ldots$ & $0 \%$ & $\ldots$ & $1 \%$ \\
\hline Amériques & 398 & $67 \%$ & $30 \%$ & $47 \%$ & $27 \%$ \\
\hline dont États-Unis & 256 & $\ldots$ & $20 \%$ & $\ldots$ & $18 \%$ \\
\hline dont Canada & 31 & $\ldots$ & $2 \%$ & $\ldots$ & $3 \%$ \\
\hline dont Mexique & 29 & $\ldots$ & $2 \%$ & ... & $1 \%$ \\
\hline Asie et Océanie & 332 & $6 \%$ & $25 \%$ & $4 \%$ & $7 \%$ \\
\hline dont Chine & 274 & $\ldots$ & $21 \%$ & ... & $1 \%$ \\
\hline Afrique & 117 & $24 \%$ & $9 \%$ & $5 \%$ & $1 \%$ \\
\hline dont Nigeria & 41 & $\ldots$ & $3 \%$ & $\ldots$ & $0 \%$ \\
\hline \begin{tabular}{|l|} 
Anglais \\
\end{tabular} & 723 & $69 \%$ & $55 \%$ & $88 \%$ & $\ldots$ \\
\hline Chinois & 240 & $4 \%$ & $18 \%$ & $2 \%$ & $\ldots$ \\
\hline Espagnol & 108 & $3 \%$ & $8 \%$ & $1 \%$ & $\ldots$ \\
\hline Français & 57 & $12 \%$ & $4 \%$ & $1 \%$ & \\
\hline Portugais & 25 & $4 \%$ & $2 \%$ & $0 \%$ & $\ldots$ \\
\hline Russe & 19 & $0 \%$ & $1 \%$ & $0 \%$ & \\
\hline Allemand & 14 & $50 \%$ & $1 \%$ & $1 \%$ & ... \\
\hline Autres langues & 43 & $2 \%$ & $3 \%$ & $0 \%$ & \\
\hline Multilingue & 81 & $44 \%$ & $6 \%$ & $6 \%$ & $\ldots$ \\
\hline Total & 1310 & $43 \%\{=565\}$ & $100 \%(=1310)$ & $100 \%$ (= 566) & $100 \%(=787)$ \\
\hline
\end{tabular}

(a) BELGEO, Biotechnologie, Agronomie, Société et Environnement (BASE), Bulletin de la Société géographique de Liège, Geologica Belgica.

Il propose, à tout le moins, que le "monde de la géographie" procède à ses évaluations sur une base plus équilibrée, par exemple inspirée de celle utilisée par l'Association européenne des Instituts de développement (EADI), qui répartit la production scientifique en 5 catégories :

1. les publications dans les journaux classés dans le tiers supérieur des facteurs d'impact de leur catégorie (en l'occurrence plus de 2,7 en géographie physique, plus de 1,8 en géographie humaine $)^{12}$;

2. les publications dans d'autres journaux cotés dans le Web of Science;

3. les publications dans des revues non cotées dans le Web of Science, mais réalisant une procédure de référencement en aveugle ;

4. les autres revues académiques ;

5. les revues non-académiques (journaux de professionnels, politiques, etc.).

33 En outre, la procédure devrait inclure les publications d'ouvrages ou de chapitres d'ouvrages, ou d'autres types de média. 


\section{Conclusions}

\section{confrontés à l'emprise hégémonique de revues spécialisées, cô̂teuses, réputé} internationales, mais de fait très largement dominées par le mode de pensée anglo-saxon. Il est difficile dans ces conditions de maintenir une littérature de qualité reflétant mieux la diversité du monde et des approches. En outre, ce biais est renforcé, en termes de facteurs d'impact bibliométriques, auxquels bien des universités croient devoir se soumettre. Les facteurs d'impact des revues spécialisées par thème, largement dominées par des non-géographes, sont en moyenne supérieurs à ceux des revues géographiques généralistes ou d'études régionales. Il en résulte une tentation à écrire dans les premières, ce qui contribue à accentuer la fragmentation et la dilution de la géographie.

Mais le combat n'est toutefois pas perdu d'avance, comme le montrent les réactions manifestées récemment par l'Union Géographique Internationale. Il peut passer entre autres par la diffusion de l'open access gratuit tant pour les auteurs que pour les lecteurs. Il impose, pour les revues qui veulent échapper à cette emprise tout en ayant une bonne visibilité, de combiner exigence, diversité et pluriculturalité, tout en tentant d'échapper aux formatages de la pensée scientifique.

Mais le passage à l'open access impose aussi de réfléchir, au risque de voir disparaître plus encore, dans le chef des étudiants et des chercheurs, une connaissance globale de l'état de la recherche géographique, à la communication du contenu de la production scientifique. Les revues généralistes sur papier permettaient de s'en faire rapidement une idée en feuilletant les tables des matières, de découvrir ainsi de nouvelles approches ou préoccupations. Aujourd'hui, la consultation est éclatée, s'effectue par mots clés sur des supports informatiques, en fonction d'objets précis et pré-déterminés de recherche. Elle n'est plus l'occasion d'une vision synthétique ou de découvertes fortuites. C'est là un risque supplémentaire pour la géographie si celle-ci se veut science intégratrice de l'analyse des structures spatiales.

\section{BIBLIOGRAPHIE}

DASSA M., KOSMOPOULOS C. \& PUMAIN D. (2010), “JournalBase. Comparer les bases de données scientifiques internationales en sciences humaines et sociales (SHS)", Cybergeo. European Journal of Geography [En ligne], Dossiers, Les revues en sciences humaines et sociales, mis en ligne le 08 janvier 2010, URL : http://cybergeo.revues.org/22864.

GINGRAS Y. (2008), La fièvre de l'évaluation de la recherche. Du mauvais usage de faux indicateurs, Note de recherche 5, Centre Interuniversitaire de recherche sur la science et la technologie, Université du Québec à Montréal, Université de Montréal, Université de Sherbrooke.

REISSE J. (2011), “L'expertise scientifique et le choix des experts en question”, Revue électronique de la Classe Technologie et Société de l'Académie Royale de Belgique. 
Sources :

AERES : Agence d'évaluation de la recherche et de l'enseignement supérieur : http://www.AERESevaluation.fr/La-liste-des-revues-scientifiques

DIRECTORY OF OPEN ACCESS JOURNALS (DOAJ): http://www.doaj.org/

JOURNALS DATABASE IGU/UGI : http://www.igu-online.org/site (LE SITE REPREND AUSSI LA PRÉSENTATION FAITE PAR TON DIETZ).

PUBLISH OR PERISH: http://www.harzing.com/pop.htm

SCOPUS : http://info.Scopus.com/detail/what/

THE WEB OF SCIENCE (WOS): http://scientific.thomson.com/mjl/

\section{ANNEXES}

\section{Les revues les mieux cotées d'après les données de Publish or Perish, par catégorie}

Les classements présentés ci-dessous illustrent, parfois de manière caricaturale, les biais évoqués dans l'article. Ainsi, si on considère, parmi les revues de notre relevé, celles publiées aux Etats-Unis, en Grande-Bretagne, en France, aux Pays-Bas ou encore en Allemagne et qui se classent parmi les cinq mieux cotées dans chacun de ces pays, aucune n'est une véritable revue de géographie ou ne contient ce mot dans son intitulé. Très souvent, le positionnement favorable des revues s'explique donc par le fait qu'elles sont fortement reconnues par d'autres disciplines, alors qu'elles ne sont que marginales pour la géographie ou en tout cas pour la grande majorité des géographes. Le H-index de revues françaises de grande qualité, comme l'Espace géographique ou Cybergeo, apparaît dérisoire et sans aucun rapport avec la valeur intrinsèque et les exigences de ces journaux. En outre, certaines revues de qualité et disposant d'un très important lectorat sont oubliées par les rankings, parce qu'elles sont destinées à un public professionnel et non à quelques chercheurs publiants spécialisés. Elles contribuent pourtant largement à la diffusion sociale de la connaissance géographique.

Le tableau ci-dessous reprend, par catégories, les 5 revues possédant les $\mathrm{H}$-index les plus élevés sur Publish or Perish. Pour les revues généralistes, le classement a été étendu aux 24 premières (H-index égaux ou supérieurs à 10). Pour les classements par pays de publication, nous avons ajouté aux cinq premières, si elles ne sont pas incluses parmi celles-ci, les deux premières revues de géographie généraliste.

\begin{tabular}{|l|l|l|}
\hline Catégories & Titres & $\begin{array}{l}\text { H-Index Publish } \\
\text { or Perish }\end{array}$ \\
\hline Revues généralistes & $\begin{array}{l}\text { Geoforum. Journal of physical, human, } \\
\text { and regional geosciences }\end{array}$ & 41 \\
\cline { 2 - 3 } & $\begin{array}{l}\text { Annals of the Association of American } \\
\text { Geographers }\end{array}$ & 37 \\
\hline
\end{tabular}




\begin{tabular}{|c|c|c|}
\hline & $\begin{array}{l}\text { Transactions of the Institute of British } \\
\text { Geographers }\end{array}$ & 34 \\
\hline & Geographical Journal & 28 \\
\hline & $\begin{array}{l}\text { Acta Geographica Sinica. Journal of } \\
\text { geographical science }\end{array}$ & 27 \\
\hline & Area & 26 \\
\hline & GeoJournal & 25 \\
\hline & Journal of Geographical Systems & 22 \\
\hline & Geographical Research & 22 \\
\hline & Geographical Analysis & 22 \\
\hline & Scripta Nova & 20 \\
\hline & Australian Geographer & 18 \\
\hline & $\begin{array}{l}\text { Journal of Geography in Higher } \\
\text { Education }\end{array}$ & 18 \\
\hline & $\begin{array}{l}\text { Singapore Journal of Tropical } \\
\text { Geography }\end{array}$ & 16 \\
\hline & $\begin{array}{l}\text { Canadian Geographer / Le géographe } \\
\text { canadien }\end{array}$ & 14 \\
\hline & Journal of Geographical Sciences & 14 \\
\hline & Journal for Geography & 14 \\
\hline & Terra & 14 \\
\hline & Journal of Geography & 14 \\
\hline & Erdkunde & 12 \\
\hline & Geografisk Tidsskrift & 12 \\
\hline & Norsk Geografisk Tidsskrift & 11 \\
\hline & Geographical Review & 11 \\
\hline & New Zealand Geographer & 10 \\
\hline Études de zones & Polis & 35 \\
\hline & European Union Politics & 33 \\
\hline
\end{tabular}




\begin{tabular}{|c|c|c|}
\hline & African Affairs & 30 \\
\hline & China Quarterly & 27 \\
\hline & Europe-Asia Studies & 24 \\
\hline \multirow{5}{*}{$\begin{array}{l}\text { Géographie politique, culturelle, sociale, } \\
\text { de la population, épistémologie et } \\
\text { histoire de la géographie }\end{array}$} & Progress in Human Geography & 46 \\
\hline & Marine Policy & 38 \\
\hline & $\begin{array}{l}\text { Journal of Ethnic and Migration } \\
\text { Studies }\end{array}$ & 35 \\
\hline & International Migration Review & 33 \\
\hline & Social and Cultural Geography & 27 \\
\hline \multirow{5}{*}{$\begin{array}{l}\text { Études urbaines, aménagement, science } \\
\text { régionale }\end{array}$} & Journal of Urban Economics & 55 \\
\hline & Landscape and Urban Planning & 52 \\
\hline & Environment and Planning A & 48 \\
\hline & Landscape Ecology & 47 \\
\hline & Urban Studies & 46 \\
\hline \multirow{5}{*}{ Géographie économique et des transports } & Energy & 61 \\
\hline & Journal of Economic Geography & 47 \\
\hline & $\begin{array}{l}\text { Transportation Research, Part B: } \\
\text { Methodological }\end{array}$ & 44 \\
\hline & $\begin{array}{l}\text { Transportation Research, Part A: } \\
\text { Policy and Practice }\end{array}$ & 42 \\
\hline & CESifo Economic Studies & 39 \\
\hline \multirow{5}{*}{$\begin{array}{l}\text { Gestion de l'environnement, monde rural, } \\
\text { développement }\end{array}$} & Development & 93 \\
\hline & $\begin{array}{l}\text { Environmental Modelling and } \\
\text { Software }\end{array}$ & 51 \\
\hline & Land Use Policy & 40 \\
\hline & $\begin{array}{l}\text { Ambio. A journal of the human } \\
\text { environment }\end{array}$ & 38 \\
\hline & Environmental Management & 37 \\
\hline Géographie du tourisme & Annals of Tourism Research & 48 \\
\hline
\end{tabular}




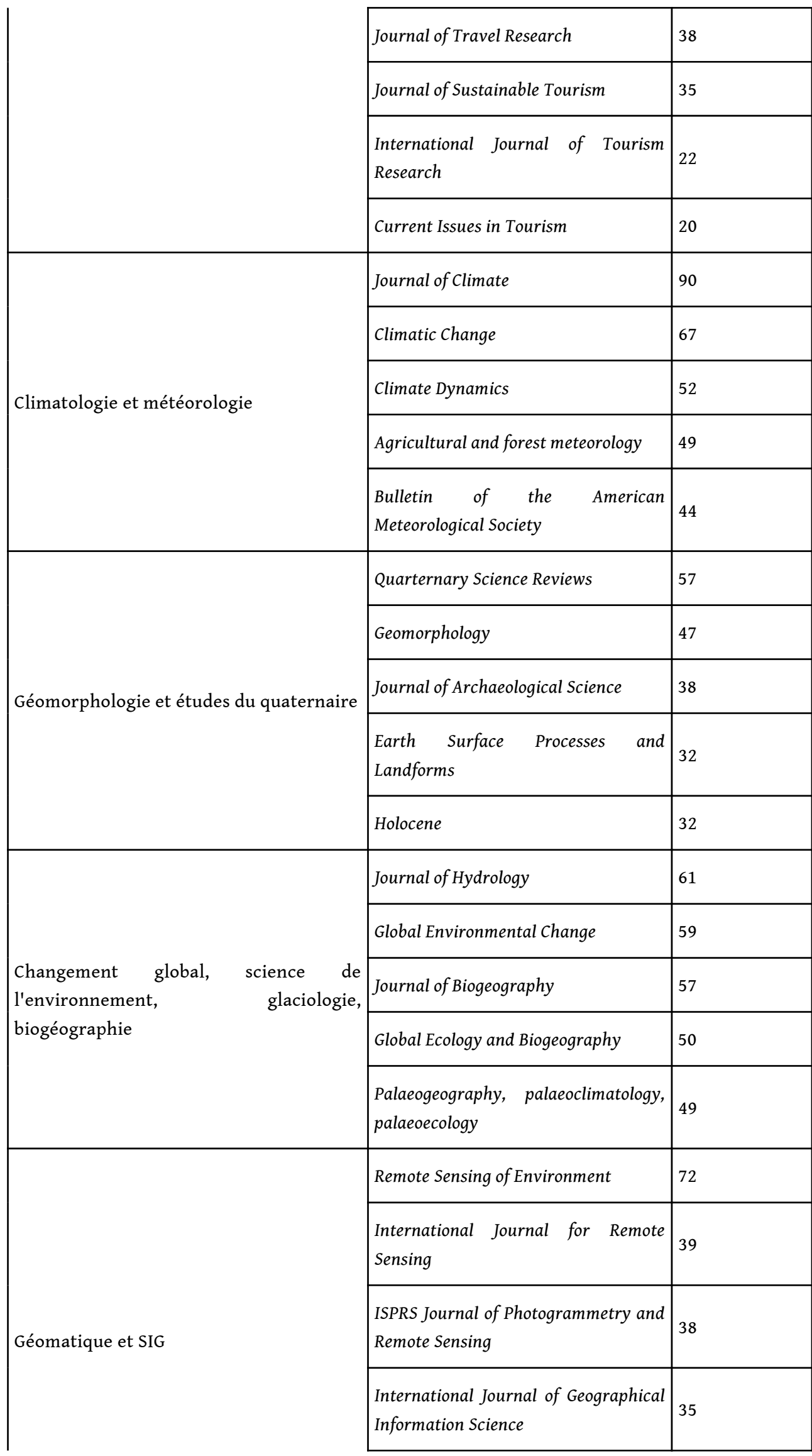




\begin{tabular}{|c|c|c|}
\hline & $\begin{array}{l}\text { Photogrammetric Engineering and } \\
\text { Remote Sensing }\end{array}$ & 35 \\
\hline \multirow{7}{*}{ Revues publiées en Grande-Bretagne } & Development & 93 \\
\hline & Quarternary Science Reviews & 57 \\
\hline & Journal of Biogeography & 57 \\
\hline & Global Ecology and Biogeography & 50 \\
\hline & Environment and Planning A & 48 \\
\hline & $\begin{array}{l}\text { [Geoforum; journal of physical, } \\
\text { human and regional geosciences] }\end{array}$ & {$[41]$} \\
\hline & $\begin{array}{l}\text { [ Transactions of the Institute of } \\
\text { British Geographers] }\end{array}$ & {$[34]$} \\
\hline \multirow{7}{*}{ Revues publiées aux Etats-Unis } & Journal of Climate & 90 \\
\hline & Journal of Urban Economics & 55 \\
\hline & Annals of Tourism Research & 48 \\
\hline & Landscape Ecology & 47 \\
\hline & $\begin{array}{l}\text { Bulletin of the American } \\
\text { Meteorological Society }\end{array}$ & 44 \\
\hline & $\begin{array}{l}\text { [Annals of the Association of American } \\
\text { Geographers] }\end{array}$ & [37] \\
\hline & [Geographical Analysis] & {$[22]$} \\
\hline \multirow{5}{*}{ Revues publiées au Canada } & $\begin{array}{l}\text { Canadian Journal of Forest Research / } \\
\text { Revue Canadienne de recherche } \\
\text { forestière }\end{array}$ & 35 \\
\hline & Arctic & 20 \\
\hline & Canadian Journal of Remote Sensing & 17 \\
\hline & $\begin{array}{l}\text { Canadian Geographer / Le géographe } \\
\text { canadien }\end{array}$ & 14 \\
\hline & $\begin{array}{l}\text { ACME. An International E-journal for } \\
\text { critical geographies }\end{array}$ & 14 \\
\hline \multirow[t]{2}{*}{ Revues publiées en France } & Population & 13 \\
\hline & Espaces et Sociétés & 11 \\
\hline
\end{tabular}




\begin{tabular}{|c|c|c|}
\hline & Développement durable et Territoires & 11 \\
\hline & Communications & 11 \\
\hline & Geodinamica Acta & 10 \\
\hline & [CyberGeo] & {$[9]$} \\
\hline & [GeoCarrefour] & {$[7]$} \\
\hline & Remote Sensing of Environment & 72 \\
\hline & Climatic Change & 67 \\
\hline & Energy & 61 \\
\hline Revues publiées aux Pays-Bas & Journal of Hydrology & 61 \\
\hline & Global Environmental Change & 59 \\
\hline & [GeoJournal] & {$[25]$} \\
\hline & [Nederlandse Geografische Studies] & {$[4]$} \\
\hline & Climate Dynamics & 52 \\
\hline & Hydrology and Earth System Sciences & 39 \\
\hline & Environmental Management & 37 \\
\hline $\begin{array}{l}\text { Revues publiées en Allemagne et en } \\
\text { Autriche }\end{array}$ & Catena & 34 \\
\hline & Theorectical and Applied Climatology & 32 \\
\hline & [Journal of Geographical Systems] & {$[22]$} \\
\hline & [Erdkunde] & {$[12]$} \\
\hline & Polis & 35 \\
\hline & Sociedade e Estado & 12 \\
\hline & Revista Brasileira de Cartografia & 10 \\
\hline Revues publiées en Amérique latine & Migraciones Internacionales & 9 \\
\hline & Boleim Goiano de Geografia & 6 \\
\hline & $\begin{array}{l}\text { [Investigaciones Geograficas. Boletin } \\
\text { de l'Instituto de Geografia de } \\
\text { l'Universidad Autonoma de Mexico] }\end{array}$ & [4] \\
\hline $\begin{array}{l}\text { Revues publiées en Europe centrale et } \\
\text { orientale }\end{array}$ & Journal for Geography & 14 \\
\hline
\end{tabular}
orientale 


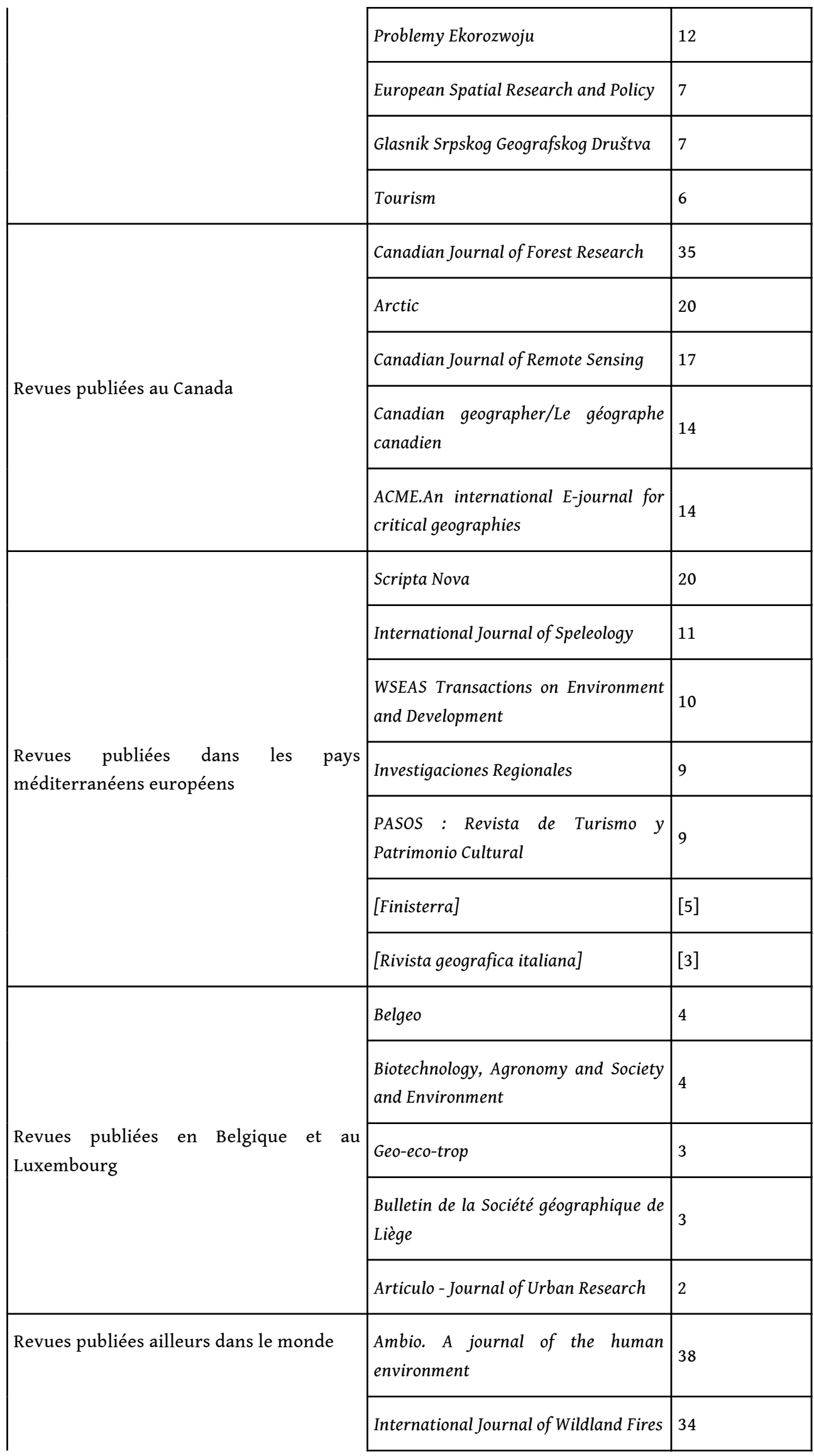




\begin{tabular}{|c|c|c|}
\hline & $\begin{array}{l}\text { Acta Geographica Sinica. Journal of } \\
\text { geographical science }\end{array}$ & 27 \\
\hline & $\begin{array}{l}\text { Geografiska Annaler, Series B: Human } \\
\text { Geography }\end{array}$ & 23 \\
\hline & Australian Geographer & 18 \\
\hline \multirow{5}{*}{$\begin{array}{l}\text { Revues des sociétés de géographie (ou } \\
\text { associations de géographes) anglo- } \\
\text { saxonnes }\end{array}$} & $\begin{array}{l}\text { Annals of the Association of American } \\
\text { Geographers }\end{array}$ & 37 \\
\hline & $\begin{array}{l}\text { Transactions of the Institute of British } \\
\text { Geographers }\end{array}$ & 34 \\
\hline & Geographical Journal & 28 \\
\hline & Area & 26 \\
\hline & $\begin{array}{l}\text { Canadian geographer/Le géographe } \\
\text { canadien }\end{array}$ & 14 \\
\hline \multirow{5}{*}{$\begin{array}{l}\text { Revues des sociétés de géographie (ou } \\
\text { associations de géographes) non-anglo- } \\
\text { saxonnes }\end{array}$} & $\begin{array}{l}\text { Geografiska Annaler, Series B: Human } \\
\text { Geography }\end{array}$ & 23 \\
\hline & Terra & 14 \\
\hline & $\begin{array}{l}\text { Geografiska Annaler, Series A: Physical } \\
\text { Geography }\end{array}$ & 13 \\
\hline & Geografisk Tidsskrift & 12 \\
\hline & Norsk Geografisk Tidsskrift & 11 \\
\hline \multirow{5}{*}{$\begin{array}{l}\text { Revues des sociétés de géographie (ou } \\
\text { associations de géographes) non-anglo- } \\
\text { saxonnes et non publiées exclusivement } \\
\text { en anglais }\end{array}$} & Die Erde & 8 \\
\hline & $\begin{array}{l}\text { Mitteilungen der Österreichischen } \\
\text { Geografische Gesellschaft }\end{array}$ & 7 \\
\hline & Glasnik Srpskog Geografskog Društva & 7 \\
\hline & Geographica helvetica & 7 \\
\hline & Geofocus & 6 \\
\hline \multirow{4}{*}{$\begin{array}{l}\text { Revues généralistes et de géographie } \\
\text { humaine en open access }\end{array}$} & Polis & 35 \\
\hline & Scripta Nova & 20 \\
\hline & Journal for Geography & 14 \\
\hline & $\begin{array}{l}\text { ACME.An international E-journal for } \\
\text { critical geographies }\end{array}$ & 14 \\
\hline
\end{tabular}




\begin{tabular}{|l|l|l|}
\cline { 2 - 3 } & Asian Development Review & 12 \\
\hline \multirow{4}{*}{$\begin{array}{l}\text { Revues de géographie physique et de } \\
\text { géomatique en open access }\end{array}$} & Hydrology and Earth System Sciences & 39 \\
\cline { 2 - 4 } & $\begin{array}{l}\text { Hydrology and Earth System Sciences. } \\
\text { Discussions }\end{array}$ & 21 \\
\cline { 2 - 4 } & Mountain Research and Development & 18 \\
\cline { 2 - 4 } & Remote Sensing & 13 \\
\cline { 2 - 4 } & International Journal of Speleology & 11 \\
\hline & & \\
\hline
\end{tabular}

\section{NOTES}

1. Ce dépouillement était clôturé au moment de la présentation par l'Union géographique internationale de son site des revues de géographie. Rester dans les délais exigés pour la publication du présent texte excluait d'intégrer cet inventaire dans l'étude, mais nous en aborderons un rapide survol en postface.

2. Dont nous avons exclu La géographie, devenue simple Bulletin de liaison des membres de la Société de géographie de Paris.

3. Open access total, ou éventuellement après deux ou trois ans d'embargo.

4. Que ce soit pour des revues en open access ou pour des revues classiques, le paiement par les auteurs pour être publié est une nouvelle forme de dérive de la marchandisation : en effet, ces montants payés par les auteurs sont de fait prélevés sur les ressources de leurs contrats de recherche ou de leurs budgets de fonctionnement. Il s'agit donc d'un transfert de fonds souvent publics soustraits du financement de la recherche au profit de maisons d'édition privées, alors qu'une saine pratique voudrait plutôt que ces maisons paient des droits d'auteur à ceux qui, par leur production, leur permettent de se développer. Quand elles ne font pas payer aux auteurs l'accès à leur propre production!

5. Nous n'avons pas considéré le classement de l'European Reference Index for Humanities (ERIH), publié par la Fondation européenne pour la Science dans l'objectif de limiter la suprématie américaine et partiellement anglo-saxonne, dans la mesure où cet organisme n'a pas (encore ?) traité le domaine de la géographie en tant que science sociale (à la différence des Life, Earth and Environmental Sciences).

6. Le site de l'Union géographique internationale recense ainsi 240 revues chinoises, alors que le Web of Science n'en reprend que 2. L'Académie des sciences chinoise a aujourd'hui, du fait de la montée en puissance économique de ce pays, un poids suffisant pour négocier une révision par le Web of Science de sa procédure de prise en considération des revues chinoises, mais ce n'est certainement pas le cas d'autres pays, en particulier les pays africains, mais aussi latinoaméricains.

7. Le H-index est la valeur égale au nombre d'articles cités au moins $\mathrm{n}$ fois, $\mathrm{n}$ étant le plus grand nombre qui corresponde à la fois au nombre d'articles cités et au nombre de leurs citations. Ainsi, si une revue a publié un article cité 50 fois, 50 articles cités 1 fois et 5 articles cités chacun 5 fois, son H-index sera de 6 ( 6 articles ont au moins été cités 5 fois). Le facteur d'impact, pour une année donnée, est le nombre moyen de citations de chaque article publié dans ce journal relevées dans les autres journaux durant la période des 2 ans précédents. Il est évident qu'il est difficile de comparer les indices bibliométriques de journaux relevant de disciplines, voire sous-disciplines, 
différentes, qui ont chacune leurs propres traditions de citation, dont la "durée de vie" des articles est très diverse et dont en outre la généralité des champs de recherche peut être très différente. Les indices bibliométriques sont aussi influencés par le nombre d'articles publiés par une revue et ils ne tiennent pas compte du contexte dans lequel sont faites les citations.

8. Le coefficient de corrélation entre les H-index Publish or Perish et ceux de SCOPUS pour ces revues est de $\mathrm{r}=0,94$; entre le H-index Publish or Perish et les facteurs d'impact du Web of Science, il est de $r=0,83$.

9. A l'intérieur de cette catégorie, les revues généralistes, de fait largement orientées vers la géographie humaine et vers les sujets les plus locaux (ce qui ne signifie pas les moins intéressants ou les moins utiles socialement) ont logiquement les scores moyens les plus bas $(6,4)$, suivies par les revues multidisciplinaires d'études de zones (études africaines, asiatiques, européennes, etc.) $(10,0)$. A l'inverse, les revues de géographie économique et des transports ont le score le plus fort, 16,8 .

10. Avec 7 revues, la Belgique et le Luxembourg sont à 3,7. Parmi ces 7 revues, 3 (dont Belgeo) sont recensées dans SCOPUS, aucune dans le Web of Science. Remarquons que Brussels Studies, qui n'est pas reprise dans notre liste, a un H-index de 7, supérieur à celui des trois revues SCOPUS, ce qui rend compte à la fois de son intérêt pour la société bruxelloise, de la traduction systématique en trois langues des travaux publiés et de l'intérêt de l'open access.

11. Ou se soumettent à leurs règles quand elles sont reprises par des maisons d'édition

12. Facteur d'impact du Web of Science. Sans doute vaudrait-il mieux un indice fondé sur la base SCOPUS, dont on a vu qu'elle recense plus largement, en particulier en sciences humaines et sociales.

\section{RÉSUMÉS}

Plus encore que d'autres sciences, du fait de l'inscription de ses recherches dans le local comme dans le global, la géographie souffre de la domination de la littérature anglo-saxonne dite internationale contrôlée par de grands groupes éditoriaux. Sur la base d'un dépouillement de 787 titres de revues géographiques et de l'analyse de leurs cotations, le biais de notoriété apparaît flagrant en faveur des revues publiées en anglais par les grands éditeurs commerciaux, sans commune mesure avec la qualité intrinsèque des publications. En outre, un biais est aussi évident en faveur des revues thématiques spécialisées, très souvent non spécifiquement géographiques, aux dépens des revues de géographie générale et des études régionales. Le recours à l'open access apparaît comme une des réponses de pays latins (France, Espagne, Brésil, Roumanie) à cette domination. Cette contribution s'inscrit pleinement dans les efforts de l'Union Géographique Internationale de promouvoir une diffusion équilibrée et diversifiée de la recherche géographique mondiale.

Yet more than for other sciences, due to researches coupling local and global, geography suffers from the domination of the "international" Anglo-Saxon literature controlled by the big publishers. On the basis of the collection of 787 geographical journals and the analysis of their rankings, a bias appears to be evident in favour of the journals published in English by the big commercial publishers, not related to the specific quality of the papers. Moreover, another bias is obvious in favour of the specialised topical journals, very often not specifically geographical ones, at the expense of the general geographical and area studies journals. The development of 
open access journals seems to be an answer of some Latin countries (France, Spain, Brazil, Romania) to this imperialistic challenge. This paper is fully on line with the endeavours of the International Geographical Union (IGU) for promoting a fair, balanced and diversified dissemination of the world geographical research.

INDEX

Mots-clés : revues de géographie, bibliométrie, classement, libre accès, Union Géographique Internationale (UGI), Web of Science, SCOPUS, Publish or Perish

Keywords : geographical journals, bibliometry, ranking, open access, International Geographical Union (IGU)

\section{AUTEUR}

\section{CHRISTIAN VANDERMOTTEN}

Université libre de Bruxelles, cvdmotte@ulb.ac.be 